\title{
Diffusive and Arrestedlike Dynamics in Currency Exchange Markets
}

\author{
J. Clara-Rahola, ${ }^{1,2, *}$ A. M. Puertas, ${ }^{1, \dagger}$ M. A. Sánchez-Granero, ${ }^{3}$ J. E. Trinidad-Segovia, ${ }^{4}$ and F. J. de las Nieves ${ }^{1}$ \\ ${ }^{1}$ Department of Applied Physics, University of Almería, 04120 Almería, Spain \\ ${ }^{2}$ i2TiC Multidisciplinary Research Group, Open University of Catalonia, 08035 Barcelona, Spain \\ ${ }^{3}$ Department of Mathematics, University of Almería, 04120 Almería, Spain \\ ${ }^{4}$ Department of Economics and Business, University of Almería, 04120 Almería, Spain
}

(Received 29 June 2016; revised manuscript received 27 October 2016; published 10 February 2017)

\begin{abstract}
This work studies the symmetry between colloidal dynamics and the dynamics of the Euro-U.S. dollar currency exchange market (EURUSD). We consider the EURUSD price in the time range between 2001 and 2015, where we find significant qualitative symmetry between fluctuation distributions from this market and the ones belonging to colloidal particles in supercooled or arrested states. In particular, we find that models used for arrested physical systems are suitable for describing the EURUSD fluctuation distributions. Whereas the corresponding mean-squared price displacement (MSPD) to the EURUSD is diffusive for all years, when focusing in selected time frames within a day, we find a two-step MSPD when the New York Stock Exchange market closes, comparable to the dynamics in supercooled systems. This is corroborated by looking at the price correlation functions and non-Gaussian parameters and can be described by the theoretical model. We discuss the origin and implications of this analogy.
\end{abstract}

DOI: 10.1103/PhysRevLett.118.068301

Slow dynamics is common to multiple physical systems such as atoms, granular, and soft-matter systems, as all of them exhibit universal features when approaching the transition towards glass or jammed states [1-7]. A particular hallmark of such a framework is the transition towards fluctuation distributions far from Gaussian, usually characterized by long tails, that depict slow structural relaxations within such arrested systems [8-13]. Different models have been proposed to reproduce these observations [14,15]. Within this regard, soft-matter systems such as colloids, polymers, or surfactants have been established as canonic in nonequilibrium systems by their own right [16,17], also exhibiting strong symmetries with many other fields, for example, with atomic or molecular systems [18-20]. Such symmetry originated because these are many-body systems of interacting particles, described by equilibrium and nonequilibrium statistical mechanics [21].

Another field that is strongly amenable to be described by statistical mechanics is financial markets [22], where statistical mechanics has proven to be a useful tool. Bachelier's Ph.D. thesis Theory of Speculation triggered, a century ago, an increasing interest for finance from a physical and mathematical point of view [23-26]. The introduction of computational techniques allowed the development of models such as the fractal one from Mandelbrot, which in fact resembles fractal descriptions also appearing in colloidal structures [27,28]. However, it has been after the works by Stanley et al. [29] when the number of research papers published by physicists, in economics in general and in finance in particular, has become relevant. Other achievements such as the generalized autoregressive conditional heteroskedasticity (GARCH) model or the Black-Scholes equation helped in the rise of the field known as econophysics, which aims to employ physical theories and models in finance [30-34]. Still, when considering a physical scope, market dynamics is not fully understood; usually, stochastic processes, statistical mechanics, or nonlinear physics are considered when describing market dynamics, but a unified body that describes the equivalence between mass or lengths with financial magnitudes is lacking.

In this Letter, we study financial markets, namely, foreign exchange markets focused on the Euro-U.S. dollar exchange rate (EURUSD), from a physical approach, typical of undercooled systems. We study the distribution of the variation of the EURUSD price and analyze it with a theoretical model of supercooled colloids, where the particles are transiently trapped but can escape on a large time scale. EURUSD dynamics is considered by computing the mean-squared price displacement (MSPD), the analog to the particle meansquared displacement (MSD). While yearly EURUSD fluctuation distributions can be described by the colloidal glass model, MSPDs are diffusive at all times, without a clear hallmark of glassy physics. Arrested states are, however, found in particular daily time frames, hallmarked by two steps in the evolution of the MSPD, the corresponding correlation function and, also, by the equivalent to the non-Gaussian parameter. The description of the dynamics of colloids and the EURUSD exchange rate poses the question of a symmetry or possible unification between both fields. Therefore, not only a descriptive approach but a physical origin to foreign exchange markets is proposed, where the EURUSD market is the analog to a supercooled colloidal system.

We have selected the EURUSD instantaneous price at time intervals of $1 \mathrm{~min}$. The price trajectory strikingly 

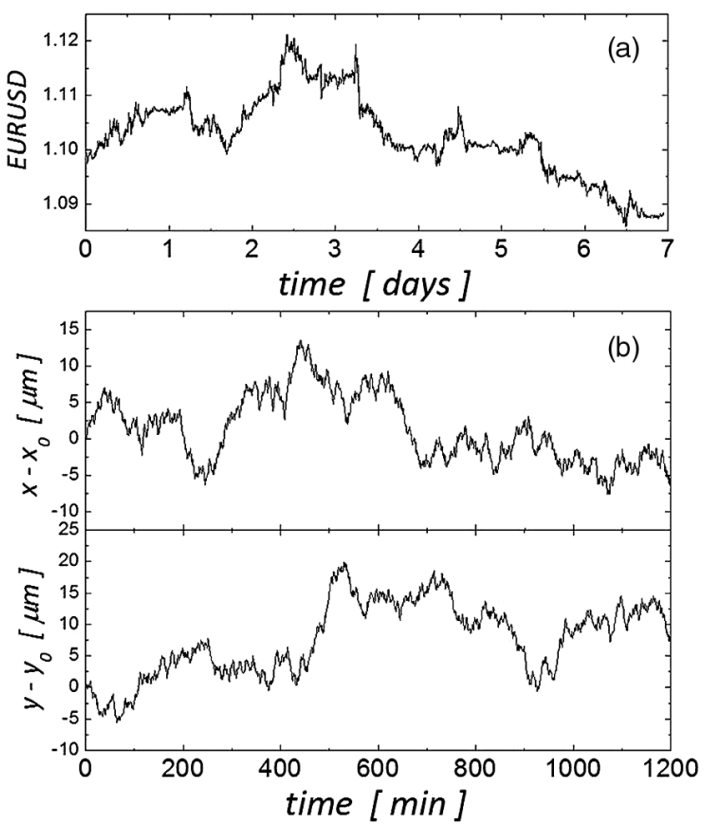

FIG. 1. Time evolution of the EURUSD price (a) and Cartesian trajectories from a colloid embedded in a quasi-two-dimensional colloidal glass (b).

resembles the trajectory of a colloidal particle in suspension, shown in Fig. 1. Intrigued by this resemblance, we wonder if the dynamics from currency exchange markets and the one from colloids exhibit a more significant symmetry and if ultimately can be described by equivalent physical laws. Note that colloidal dynamics is driven by parameters such as interparticle interactions, density, or applied shear [35-38], whereas market dynamics is determined by parameters of a very different nature, such as the trading volume, number of investors at a given period, or balance between supply and demand [39-41].

We first study price fluctuations in the EURUSD market, i.e., the difference between prices separated by a lag time $\tau$, $\delta p(\tau)=\left\langle p\left(t_{0}+\tau\right)-p\left(t_{0}\right)\right\rangle$, where the brackets indicate averaging over different time origins $t_{0}[42]$. By fixing the magnitude of $\tau$, we construct the probability distribution function (PDF) of $\delta p(\tau)$, an analog to the standard particle displacement distribution. Figure 2 shows the PDF considering a total time period from 2010 up to 2015, with $\tau=5,25,125,625$, and $3125 \mathrm{~min}$, shifted vertically for clarity. All PDFs exhibit a symmetric profile featured by long tails at large $|\delta p(\tau)|$, except at the largest $\tau$, where the PDF is Gaussian within the statistical noise. Similar tailed PDFs have been observed in other price fluctuations in finance [26,33]. These PDFs are characteristic of the particular system under study, the EURUSD in our case, and it does not change if different periods are studied. Figure 2(a) compares the PDF for the years 2001 and 2007 (years with particular economic evolution), with the average in the period 2010-2015.

Aiming to obtain a quantitative description of the price PDF, we borrow a model from glasses that has proven
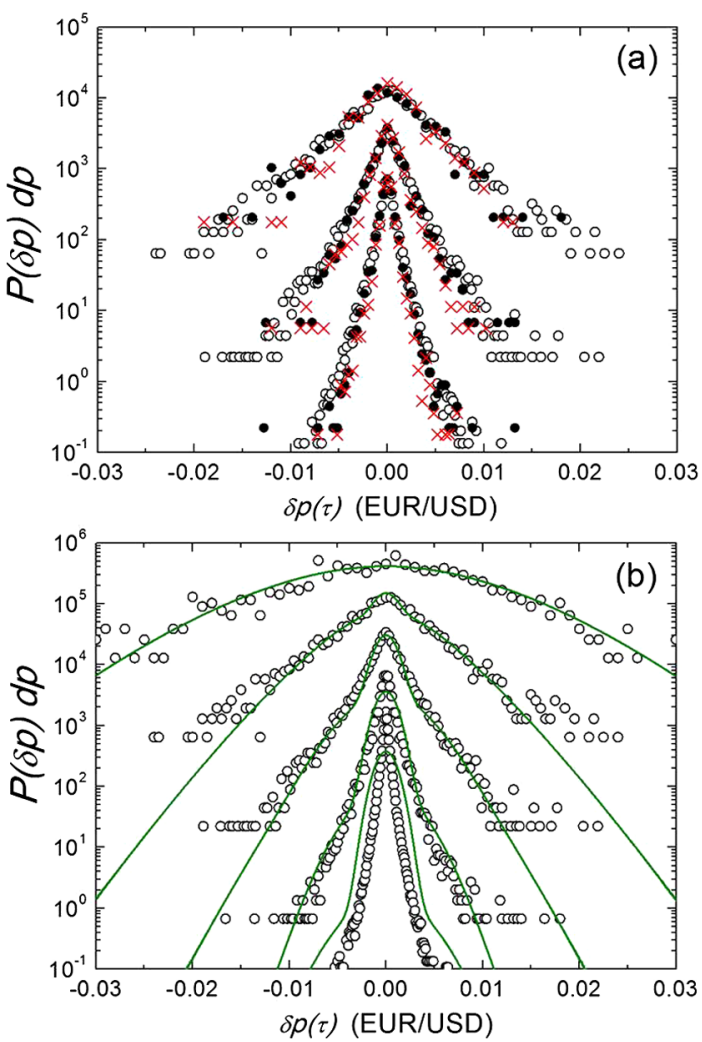

FIG. 2. EURUSD fluctuation distributions, $\delta(\tau)$ (a), for 20102015 (open circles), 2001 (solid circles), and 2007 (crosses). From bottom to top, $\tau=25,125$, and $625 \mathrm{~min}$ (shifted vertically for clarity). Fit to the experimental PDF (b) over the period 20102015 with the van Hove function of Eq. (1) (lines). From bottom to top, $\tau=5,25,125,625$, and $3125 \mathrm{~min}$ (shifted).

successful when describing data from experiments and simulations [14]. Typically, tailed distributions in economy are approached by self-similarity or described through Levy flights combined with Gaussian ones $[33,43]$, which are employed in few models, in particular, the ContBouchaud spin model [44] or the power law one [45]. However, these require heuristic arguments or restrictions, such as a constant number of market investors or agents correlated according to trading strategies, proposed to be equal to spin domains. Our scope avoids considering these kind of conditions, as we directly study the EURUSD market as a whole, where any agent can behave freely. In physical glasses, every particle is ideally caged by its own neighbors, restricting the structural relaxation of the whole system. Thermal fluctuations, however, allow particles to jump from one cage to another, on a large time scale. It is also useful to recall the classical description of glasses or undercooled fluids in terms of their free energy landscape containing multiple shallow minima (basins), separated by high barriers [46]. A similar model for financial markets, implying that the price is transiently trapped and eventually jumps out on large time scales, is attempted in the following, based on a simple model for particle glasses developed by Chaudhuri, Berthier, and Kob [14]. 
In the model, particles are transiently trapped in cages, described by a time-independent Gaussian PDF $\left[f_{\text {vib }}(r)=\left(2 \pi l^{2}\right)^{-3 / 2} \exp \left(-r^{2} / 2 l^{2}\right)\right.$ in their work]. Longrange jumps are possible, according to a Gaussian distribution $f_{\text {jump }}(r)=\left(2 \pi d^{2}\right)^{-3 / 2} \exp \left(-r^{2} / 2 d^{2}\right)$, on a large time scale. The probability to jump for the first time is given by an exponential distribution $\phi_{1}(t)=$ $\tau_{1}^{-1} \exp \left(-t / \tau_{1}\right)$, while subsequent jumps occur faster according to $\phi_{2}(t)=\tau_{2}^{-1} \exp \left(-t / \tau_{2}\right)$, with $\tau_{2}<\tau_{1}$. The overall displacement distribution, or van Hove function, $G(r, t)$ depicts the probability of finding a particle in $r$, at time $t$, and it is calculated in the Fourier-Laplace domain $G(q, s)$. Here, we have considered this van Hove distribution to analyze the EURUSD PDF, taking into consideration the new dimensionality of the problem, the scalar price instead of the position vector. Backtransforming to the price-time domain, the distribution reads

$$
\begin{aligned}
G(p, t)= & \tau_{1} f_{\mathrm{vib}}(p) \phi_{1}(t)+\mathrm{FT}^{-1}\left(\tilde{f}_{\mathrm{vib}}(q) \tilde{f}(q) \tau_{2}\right. \\
& \left.\times \frac{\exp \left\{[\tilde{f}(q)-1] t / \tau_{2}\right\}-\exp \left(-t / \tau_{1}\right)}{\tau_{2}-\tau_{1}+\tilde{f}(q) \tau_{1}}\right) .
\end{aligned}
$$

Here $\tilde{f}(q)=\tilde{f}_{\text {vib }}(q) \tilde{f}_{\text {jump }}(q), \tilde{f}(q)$ is the Fourier transform of function $f(p), q$ is the conjugate variable of price $p$ in the Fourier space, and $\mathrm{FT}^{-1}$ denotes the inverse Fourier transform. In this original model, the particle is assumed to explore its cage on a time scale much shorter than $\tau_{1}$ or $\tau_{2}$; thus, $f_{\text {vib }}(r)$ is time independent. Because this assumption cannot be made a priori in the EURUSD system, the model is modified to introduce short time diffusion, implying thus a finite time to explore the cage. For this purpose, we consider an Ornstein-Uhlenbeck process to calculate $f_{\text {vib }}(p, t)=$ $\sqrt{\alpha / 2 \pi D\left(1-e^{-2 \alpha t)}\right)} \exp \left\{-\alpha p^{2} / 2 D\left(1-e^{-2 \alpha t}\right)\right\}$, with $D$ the diffusion coefficient and $\alpha=D / l^{2}$ [47-49]. This depicts a particle describing Brownian motion with a linear central force pulling it towards its origin.

Using this new model, the experimental PDFs are fitted, as shown in Fig. 2 (lower panel), using $D, l, d, \tau_{1}$, and $\tau_{2}$ as fitting parameters, identical for all values of $\tau$. The values of the parameters are $D=2 \times 10^{-8} \mathrm{~min}^{-1}, l=30 \times 10^{-4}, d=15 \times$ $10^{-4}, \tau_{1}=400 \mathrm{~min}$, and $\tau_{2}=300 \mathrm{~min}$, resulting in very good agreement with the experimental data, except for the lowest $\tau$. The comparison of the complementary cumulative distribution functions (CDFs) from the model and experimental data, which is focused on the behavior at long distances, is also satisfactory, as shown in Supplemental Material [50]. Note that the parameters indicate the EURUSD price caged within intervals of ca. 0.30 cents, and jumps out of this range occur on a time scale of approximately $5 \mathrm{~h}$.

A hallmark of undercooled systems, as mentioned previously, is the separation between microscopic and structural dynamics, which results in correlation functions decaying in two steps or intermediate plateaus in the tagged particle MSD [57-59]. Analogously, we propose the MSPD:

$$
\left\langle\Delta p^{2}(\tau)\right\rangle=\left\langle\left[p\left(t_{0}+\tau\right)-p\left(t_{0}\right)\right]^{2}\right\rangle .
$$

Despite fluctuation PDFs being characteristic to arrested systems, the MSPD is linear, as indicated in Fig. 3. Even more, such diffusive behavior is found in all years, shown in Fig. 3, and all MSPDs exhibit a common origin, indicating that the diffusion coefficient is constant; therefore, market dynamics is characteristic of the EURUSD regardless of the year considered, including years of conflict to economy, such as 2001 or 2007. In passing, we note that the short time dynamics is Brownian, which leads us to establish the analogy with undercooled colloids, either glasses or gels, rather than atomic glasses. The MSPD calculated from our model is also presented in Fig. 3, correctly reproducing the data. The precise combination of the parameters $\left\{l, d, \tau_{1}, \tau_{2}\right\}$ and $D$ produces this linear evolution of $\Delta p^{2}(\tau)$, although the PDF differ clearly from Gaussian.

Given this unexpected result, we seek a particular case or regime where the non-Gaussian components are important enough to produce deviations in the MSPD with respect to Brownian diffusion. Thus, we deaggregate the data according to the commencing time and study the dynamics for the next $24 \mathrm{~h}$. Additionally, we change the database to secondresolved prices, to access the short time dynamics. In particular, the year 2015 is studied, and we first set the starting point for the calculation of $\left\langle\delta p^{2}(\tau)\right\rangle$ at the opening of the New York Stock Exchange (NYSE), 9:30 a.m. Eastern Time (ET). The MSPD for the forthcoming $24 \mathrm{~h}$ after this commencing time $t_{0}$ is averaged over all days. The same procedure is repeated for different values of $t_{0}$, covering the range of $24 \mathrm{~h}$. Recall that foreign exchange markets remain open continuously besides weekends. Results are presented in Fig. 4, where a striking dependence of the MSPD with $t_{0}$ can be identified.

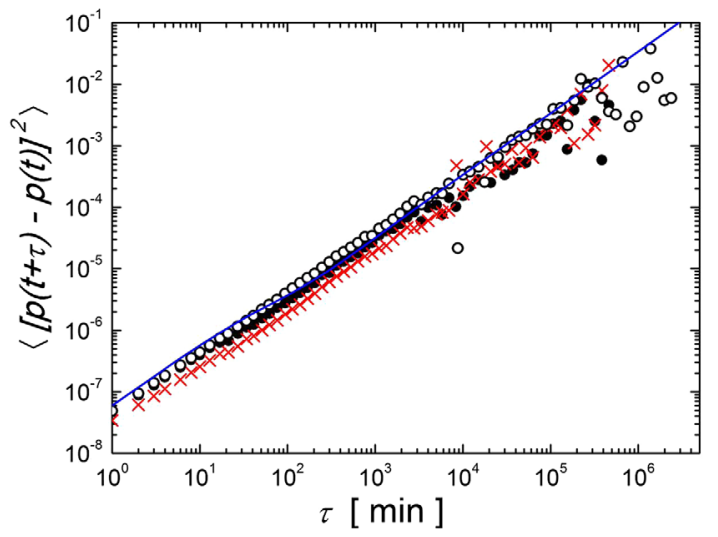

FIG. 3. EURUSD MSPDs as calculated from 1 min experimental data for the period 2010-2015 (open circles), to the year 2001 (solid circles), and to the year 2007 (crosses). The line is the MSPD according to the van Hove function of Eq. (1), with the same parameters as Fig. 2(b). 
When the opening of the NYSE is taken as the starting time, the dynamics is diffusive, but for later commencing times, the magnitude of the MSPD decreases and a shoulder appears at intermediate times, when $t_{0}$ approaches the closure of the NYSE. This trend continues developing, and at $t_{0}=6: 00$ p.m. ET the MSPD becomes minimal, exhibiting an initial diffusionlike increase at low $\tau$, crossing over to a quasiplateau, and recovering again the linear behavior for large $\tau$. This behavior is close to the profile commonly found in arrested colloidal systems, either by aggregation or at a high particle density $[60,61]$, due to the transient trapping of particles inside cages of neighbors (in glasses), or in a network of bonds (in gels). The same behavior in the MSPD suggests that price dynamics considered at daily periods with the starting time between 3:00 and 6:00 p.m. ET undergoes a temporary dynamic arrest. The transient trapping has a typical time scale of about $\tau=30 \mathrm{~min}$ and is followed by diffusive dynamics.
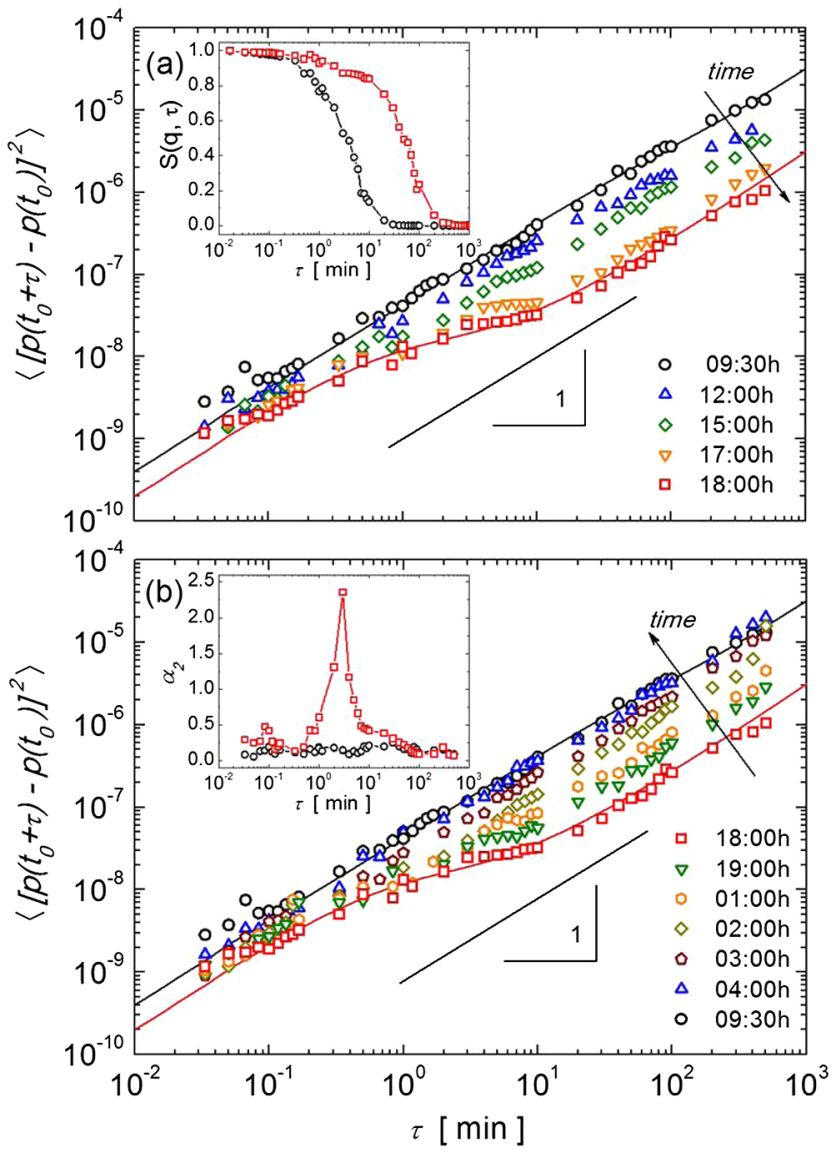

FIG. 4. EURUSD MSPD as calculated from 2015 experimental data by considering time periods of $24 \mathrm{~h}(\mathrm{ET})$. In the time range 9:30 a.m.-6:00 p.m. (a), a transition from diffusive to an undercooled dynamics is observed. Commencing times are labeled. By contrast, in the time frame of 6:00 p.m.9:30 a.m., the transition is reversed (b). The lines are the fitting of the model for the different cases. The insets show the price correlation function $S(q, \tau)$ (a) and non-Gaussian parameter $\alpha_{2}$ (b).
Commencing times later than 6:00 p.m. reverse this trend; MSPDs increase and the intermediate plateau vanishes, approaching diffusion when the starting point of the MSPD calculation is between 2:00 and 4:00 a.m., with the maximum MSPD at 9:30 a.m. This observation is consistent with the correlation of market behavior with activity, as done by Ito and Hashimoto for the U.S. dollar-Japanese yen market [62].

The theoretical model can rationalize this behavior, as shown in Fig. 4, for the two extreme cases $t_{0}=9: 30$ a.m. and 6:00 p.m. To fit the model, $D$ and the time scales $\tau_{1}$ and $\tau_{2}$ were fixed for all $t_{0}$, as these are expected to be intrinsic to the EURUSD system, and only $l$ and $d$ are varied. The fits shown in the figure are obtained for $l=3 \times 10^{-3}$ and $d=1.5 \times 10^{-3}$ for $t_{0}=9: 30$ a.m. and $l=0.1 \times 10^{-3}$ and $d=0.15 \times 10^{-3}$ for $t_{0}=6: 00$ p.m. Note that, in the latter, both $l$ and $d$ are much smaller, but also $l<d$, characteristic of arrested systems, and the cage size is of the order of $l$.

Given this similarity at the level of the MSPD, we study other properties which serve to identify undercooled states or glasses, in particular, the dynamic structure factor $S(q, \tau)=\left\langle\exp \left\{i q\left[p\left(t_{0}+\tau\right)-p\left(t_{0}\right)\right]\right\}\right\rangle$ and the one-dimensional non-Gaussian parameter $\alpha_{2}(\tau)=$ $\left\langle\Delta p(\tau)^{4}\right\rangle / 3\left\langle\Delta p(\tau)^{2}\right\rangle^{2}-1 . S(q, \tau)$ starts from 1 decaying to zero in fluids and to a finite value in glasses. In undercooled systems, it shows an intermediate plateau that depends on the wave vector $q$. For the EURUSD system, we select a value of $q \sim 2 \pi / l$ to probe this range of price variations, and the resulting correlation functions are shown in the inset in Fig. 4(a) and further detailed in the Supplemental [50]. The non-Gaussian parameter $\alpha_{2}$, on the other hand, quantifies the deviation of the PDF from Gaussian. In undercooled fluids, $\alpha_{2}$ starts from zero (the PDF is Gaussian at short times) and describes a maximum when the particles are caged and start to break free, to become zero again at long times. The non-Gaussian parameter for the EURUSD system, shown in the inset in Fig. 4(b) and detailed in Supplemental Material [50], indeed shows a maximum when $t_{0}=6: 00 \mathrm{p} . \mathrm{m}$. in the time range where the shoulder of the MSPD appears. Both $S(q, t)$ and $\alpha_{2}$ confirm the analogy between the dynamics of supercooled fluids and the EURUSD market.

The dynamics of economic markets is often described as diffusive, based on the linear MSPD profile when considering long periods, where the efficient market hypothesis $(\mathrm{EMH})$ or fractal market hypothesis (FMH) can be invoked to explain such behavior $[63,64]$. The EMH states that market prices are due to all market information being available, making it impossible to beat the market; such a statement is in fact compatible with a random walk. We show in this study that this diffusionlike behavior is found only when averages over long times are taken in the MSPD, $\left\langle\Delta p^{2}(\tau)\right\rangle$, but disappear when local time averages at $24 \mathrm{~h}$ periods are performed and where either diffusive or arrested dynamics are featured. The FMH states, on the other hand, 
that investment strategies converge when considering short time frames, arresting the market dynamics and making it more inefficient. Our analysis suggests a fluid-to-glass transition upon the choice of the reference MSPD time, implying that markets become either efficient or fractallike, depending on their activity.

In particle systems, the different dynamical regimes typical of fluid-to-glass transitions are obtained by switching a physical parameter that controls the interaction between particles. It must be then further sought which is the origin of the nontrivial dynamics shown above and if the analogy can be exploited to identify the equivalent set of parameters that control market dynamics, such as traded volume, number of investors in the market, or even policy decisions from regulatory institutions. Furthermore, the symmetry between colloidal systems and the EURUSD exchange market is as well found in other currency pairs, such as the Euro-Swiss Franc (EURCHF). A new approach appears, where exchange rate currency pairs can be regarded as colloidal systems, and, due to their coupled dynamics, the whole foreign exchange market can be considered a single undercooled system. This view and its natural extension to stock and other markets looks promising when modeling financial markets and needs to be further addressed.

We acknowledge histdata.com and oanda.com for providing all currency exchange data. Funding from UOC, Project No. N11-6139473, aimed at enhancing submission to H2020 calls (J. C.-R.), the Spanish Ministerio de Economía, Industria y Competitividad, and the European Regional Development Fund (ERDF) under Project No. FIS2015-69022-P and MTM2015-64373-P are gratefully acknowledged.

*jclarar@uoc.edu †apuertas@ual.es

[1] M. T. Dove, Structure and Dynamics: An Atomic View of Materials (Oxford University, New York, 2003).

[2] Advances in Atomic, Molecular, and Optical Physics, edited by E. Arimondo, C. C. Lin, and S. F. Yelin (Academic, New York, 2015).

[3] V. M. Giordano and B. Ruta, Nat. Commun. 7, 10344 (2016).

[4] Handbook of Granular Materials, edited by S. V. Franklin and M. D. Shattuck (CRC Press, Boca Raton, FL, 2015).

[5] C. Josserand, A. V. Tkachenko, D. M. Mueth, and H. M. Jaeger, Phys. Rev. Lett. 85, 3632 (2000).

[6] Jamming and Rheology, edited by A. J. Liu and S. R. Nagel (CRC Press, Boca Raton, FL, 2001).

[7] L. Cipelletti and L. Ramos, Curr. Opin. Colloid Interface Sci. 7, 228 (2002).

[8] P. G. N. de Vegvar and T. A. Fulton, Phys. Rev. Lett. 71, 3537 (1993).

[9] H. E. Castillo and A. Parsaeian, Nat. Phys. 3, 26 (2007).
[10] D. Rygbyt and R.J. Roe, Macromolecules 23, 5312 (1990).

[11] E. Gardel, E. Sitaridou, K. Facto, E. Keene, K. Hattam, N. Easwar, and N. Menon, Phil. Trans. R. Soc. A 367, 5109 (2009).

[12] A. Duri, H. Bissig, V. Trappe, and L. Cipelletti, Phys. Rev. E 72, 051401 (2005).

[13] D. A. Sessoms, H. Bissig, A. Duri, L. Cipelletti, and V. Trappe, Soft Matter 6, 3030 (2010).

[14] P. Chaudhuri, L. Berthier, and W. Kob, Phys. Rev. Lett. 99, 060604 (2007).

[15] N. H. March and M. P. Tosi, Atomic Dynamics in Liquids (Dover, New York, 1991).

[16] Fluids, Colloids and Soft Materials: An Introduction to Soft Matter Physics, edited by A. Fernandez-Nieves and A. M. Puertas (Wiley, New York, 2016).

[17] R. A. L. Jones, Soft Condensed Matter (Oxford University, New York, 2002).

[18] P. N. Pusey and W. van Megen, Nature (London) 320, 340 (1986).

[19] J. C. Crocker and D. G. Grier, Phys. Rev. Lett. 73, 352 (1994).

[20] K. N. Pham, A. M. Puertas, J. Berenholtz, S. U. Egelhaaf, A. Moussad, P. N. Pusey, A. B. Schofield, M. E. Cates, M. Fuchs, and W. C. K. Poon, Science 296, 104 (2002).

[21] W. Poon, Science 304, 830 (2004).

[22] Complex Systems in Finance and Econometrics, edited by R. A. Meyers (Springer, New York, 2011).

[23] L. Bachelier, Theory of Speculation (Princeton University, Princeton, NJ, 2006).

[24] E. F. Fama and M. H. Miller, The Theory of Finance (Holt, Rinehart and Winston, New York, 1972).

[25] E. F. Fama, Foundations of Finance, Portfolio Decisions and Securities Prices (Basic Books, New York, 1976).

[26] J.-P. Bouchaud and M. Potters, Theory of Financial Risks (Cambridge University Press, Cambridge, England, 2000).

[27] B. B. Mandelbrot, Fractals and Scaling in Finance (Springer, New York, 1997).

[28] E. E. Peters, Fractal Market Analysis (Wiley, New York, 1996).

[29] H. Stanley, V. Afanasyev, L. A. N. Amaral, S. Buldyrev, A. Goldberger, S. Havlin, H. Leschhorn, P. Maass, R. Mantegna, C.-K. Peng, P. A. Prince, M. A. Salinger, M. Stanley, and G. Viswanathan, Physica (Amsterdam) 224A, 302 (1996).

[30] F. Black and M. Scholes, J. Polit. Econ. 81, 637 (1973).

[31] R. N. Mantegna and H. E. Stanley, Nature (London) 383, 587 (1996).

[32] C. Francq and J.-M. Zakolan, GARCH Models: Structure, Statistical Inference and Financial Applications (Wiley, New York, 2010).

[33] R. N. Mantegna and H. E. Stanley, Introduction to Econophysics: Correlations and Complexity in Finance (Cambridge University Press, Cambridge, England, 2007).

[34] P. Richmond, J. Mimkes, and S. Hutzler, Econophysics and Physical Economics (Oxford University, New York, 2013).

[35] A. M. Puertas, M. Fuchs, and M. E. Cates, Phys. Rev. Lett. 88, 098301 (2002). 
[36] A. M. Puertas, M. Fuchs, and M. E. Cates, Phys. Rev. E 75, 031401 (2007).

[37] J. Clara-Rahola, R. Contreras-Caceres, B. Sierra-Martin, A. Maldonado-Valdivia, M. Hund, A. Fery, T. Hellweg, and A. Fernandez-Barbero, Colloids Surf. A 463, 18 (2014).

[38] J. Clara-Rahola, T. A. Brzinski, D. Semwogerere, K. Feitosa, J. C. Crocker, J. Sato, V. Breedveld, and E. R. Weeks, Phys. Rev. E 91, 010301 (2015).

[39] T. Kaizoji, S. Bornholdt, and Y. Fujiwara, Physica (Amsterdam) 316A, 441 (2002).

[40] S. M. Duarte-Queiros, Chaos Solitons Fractals 88, 24 (2016).

[41] D. Stosic, D. Stosic, T. Ludermir, W. de Oliveira, and T. Stosic, Physica (Amsterdam) 449A, 233 (2016).

[42] Note that, in stock markets, the log return is usually studied, $r(\tau)=\left\langle\ln p\left(t_{0}+\tau\right) / p\left(t_{0}\right)\right\rangle$, to account for exponential growth or a decrease of $p(t)$. In our case, however, the variation of $p(t)$ is small, yielding $r(\tau) \approx \delta p(\tau) / p\left(t_{0}\right)$.

[43] M. A. Sanchez, J. E. Trinidad, J. Garcia, and M. Fernandez, PLoS One 10, e0127824 (2015).

[44] R. Cont and J.-P. Bouchaud, Macroecon. Dyn. 4, 170 (2000).

[45] A. Clauset, C. R. Shalizi, and M. E. Newman, SIAM Rev. 51, 661 (2009).

[46] M. Goldstein, J. Chem. Phys. 51, 3728 (1969).

[47] G. E. Uhlenbeck and L. S. Ornstein, Phys. Rev. 36, 823 (1930).

[48] D. T. Gillespie, Phys. Rev. E 54, 2084 (1996).

[49] H. Risken, The FokkerPlanck Equation: Method of Solution and Applications (Springer-Verlag, New York, 1989).
[50] See Supplemental Material at http://link.aps.org/ supplemental/10.1103/PhysRevLett.118.068301, which includes Refs. [51-56], for details concerning complementary CDFs, non-Gaussian parameters, correlation functions, and the fit to MSPDs according to the theoretical model proposed in this work.

[51] A Practical Guide to Heavy Tails, edited by R. J. Adler, R. E. Feldman, and M. S. Taqqu (Birkhauser, Boston, 1998).

[52] K. Binder and W. Kob, Glassy Materials and Disordered Solids: An Introduction to Their Statistical Mechanics (revised edition) (World Scientific, Singapore, 2011).

[53] L. Cipelletti, S. Manley, R. C. Ball, and D. A. Weitz, Phys. Rev. Lett. 84, 2275 (2000).

[54] A. Rahman, Phys. Rev. 136, A405 (1964).

[55] W. Kob, C. Donati, S. J. Plimpton, P. H. Poole, and S. C. Glotzer, Phys. Rev. Lett. 79, 2827 (1997).

[56] E. R. Weeks and D. A. Weitz, Chem. Phys. 284, 361 (2002).

[57] A. Lubelski, I. M. Sokolov, and J. Klafter, Phys. Rev. Lett. 100, 250602 (2008)

[58] F. Scheffold, S. E. Skipetrov, S. Romer, and P. Schurtenberger, Phys. Rev. E 63, 061404 (2001).

[59] D. A. Weitz and D. J. Pine, Dynamic Light Scattering (Oxford University, New York, 1993).

[60] H. M. Wyss, S. Romer, F. Scheffold, P. Schurtenberger, and L. J. Gauckler, J. Colloid Interface Sci. 241, 89 (2001).

[61] C. R. Nugent, K. V. Edmond, H. N. Patel, and E. R. Weeks, Phys. Rev. Lett. 99, 025702 (2007).

[62] T. Ito and Y. Hashimoto, J. Jpn. Int. Econ. 20, 637 (2006).

[63] B. G. Malkiel and E. F. Fama, J. Finance 25, 383 (1970).

[64] E. E. Peters, Financ. Anal. J. 45, 32 (1989). 